\title{
5 Meanings of the unmarked: how 'default' person reference does more than just refer
}

\author{
N. J. Enfield
}

It's not that somebody is ordinary, it's perhaps that that's what their business is. And it takes work, as any other business does. (Harvey Sacks, Spring 1970, Lecture 1)

The aspects of things that are most important for us are hidden because of their simplicity and familiarity. (One is unable to notice something - because it is always before one's eyes.) The real foundations of his enquiry do not strike a man at all. Unless that fact has at some time struck him. - And this means: we fail to be struck by what, once seen, is most striking and most powerful. (Ludwig Wittgenstein, 1953, Philosophical Investigations $\$ 1.129$ )

This chapter explores one way in which members of a culture work to achieve the appearance of ordinariness, and in so doing render invisible their most heartfelt concerns. The target locus of behaviour is referential practice, in particular verbal reference to persons in everyday conversation. In this domain, as in any other, to be or act ordinary is to attract no special attention to that way of being or acting. For instance, by dressing in overalls, a plumber at work chooses the default, unmarked course of action. He will not be sanctioned or even commented upon for doing so (unlike, say, were he to wear a dress). When we follow a default course of action in this way, we are in one sense not doing anything special; indeed we may be taken not to be 'doing' anything at all. But since it takes work to pull off the invisible appearance of ordinariness, even when our manners of behaviour are rendered literally unremarkable by their conformity, we are nevertheless always doing something by choosing just those manners of behaviour. Along these lines, this chapter argues that default practices of person reference do more than just refer. They instantiate and stabilize culture-specific views of the person. But by their very design, these practices render their own meaning difficult to detect, shrouded in the veil of ordinariness.

In the use and interpretation of language, one key mechanism turns on a distinction between the ordinary and the exceptional. This is the contrast between default and marked formulation of spoken utterances. A default referential formulation is a way of phrasing reference (e.g., to a person, 
place, thing) that will be taken to be the standard, nothing-special-intended way of saying it (e.g., New York as opposed to The Big Apple, John as opposed to His Majesty, the dog as opposed to the hound). As the term suggests, a default formulation will represent a path of least cognitive resistance for both the production and comprehension of a referential expression. The term default implies that for a speaker the formulation is virtually automatic, in the absence of any special consideration to selection of that manner of formulation as opposed to some other conceivable one (hence the Prague School's term automatization; Hanks 1990; Havránek 1964/1932:9). It's the format you pick when you don't have any special reason to care how the thing you want to say gets said. Given the perhaps infinite number of possible ways of formulating an utterance, the existence of defaults for usage minimizes processing by providing a single, ever-present opt-out. By contrast, a marked (that is, pragmatically marked) ${ }^{1}$ referential formulation is defined as an expressive departure from the default (e.g., his majesty instead of John or he). Its foregrounded nature serves to alert the listener's attention (Hanks 1990; Havránek 1964/1932:10). By producing an 'unusual' formulation, a speaker displays some special effort, implying their having selected just that formulation for a reason, thereby inviting the listener to wonder why, and eliciting an enriched interpretation (Grice 1975, 1989; Levinson 2000).

In their seminal analyses of person reference in English conversation, Sacks and Schegloff (1979, this volume; cf. Schegloff 1996a) rely crucially on this logic of informational contrast between default versus marked manners of referential formulation. Because the default manner of formulation attracts no special attention or enriched interpretation, it is argued to be making literally no contribution to the interaction apart from establishing reference to an individual person. By contrast, with a marked formulation, 'something else in addition to referring is being done' (Schegloff 1996a: 439), above and beyond the mere informational imperatives of communication (e.g., with his majesty, I am not just referring to John, but may also be complaining about him; cf. Stivers, this volume). But while a default formulation may not be doing any special, foregrounded or topical communicative work in an interaction, I argue that it may be systematically doing more than merely achieving reference. The evidence comes from the system of person reference in Lao, a Southwestern Tai language of Laos,

${ }^{1}$ Haspelmath (2006) advises against any use of the term 'marked' due to its long list of different meanings and uses in the literature (cf. Zwicky 1978; Gair 1988, inter alia). He suggests that all relevant senses of marked are handled by independently existing terms/concepts. I appreciate the need for care, but nevertheless find the term handy. See the introduction to this book for further discussion of defaults, markedness, and the like. 
Thailand, and Cambodia. Data are from video recordings of natural conversation.

In Lao, default forms of person reference explicitly encode kin-based and other hierarchical social relations between speakers and person referents. As in many other languages (see chapters in this volume), these default formats for person reference publicize key cultural values every time they are used. By giving off information about relative social positioning, these habitual personreference formats display speakers' commitments to socially generalized values, and through this help in reproducing, maintaining and stabilizing those values. Attention to the details of linguistic structure and its deployment in face-to-face interaction illuminates questions at the heart of social anthropology. In the moment-by-moment flow of human sociality, communication constitutes social action on multiple levels, not only in the foreground of attention, but also in the disattended, quietly purring background machinery of a culture's practiced norms.

\subsection{The Lao system of person reference}

To refer to a person in conversation, a Lao speaker has many possible alternative formulations to choose from. The complexities of this set of alternatives concern distinctions of social hierarchy, as defined by (classificatory) kinship and other factors that determine relative position of individuals in social structure.

First a point of clarification about the social domain of focus in this chapter. There is significant diversity in the range of social situations a Laospeaking individual will find themselves in, and this uneven social - interactional landscape is rapidly evolving in a new, modern world (cf. Evans 1999, 2002; Rehbein 2004). Individuals have to apply and negotiate multiple distinct sets of conventions for communication, both ritual and mundane, depending on context. These important complexities are, however, beyond the scope of this chapter. For the argument presented here, I restrict the analysis to maximally informal conversations in village settings. This may be considered the basic, primordial setting for human interaction for most Lao speakers. It approximates a general standard for informal interaction in the home and family environment, and as such represents the typical setting for early socialization, and thus the base from which other, marked types of social - interactional arrangement may be considered departures. It is the common interactional standard in which the generic attitudes of Mead's (1934) 'generalized other' are grounded. In more formal or otherwise constrained situation types, these underlying interactional norms - for example, the conventions of person reference - may be manipulated for special effect. Aspects of Lao that express social - hierarchical distinctions are a site of 


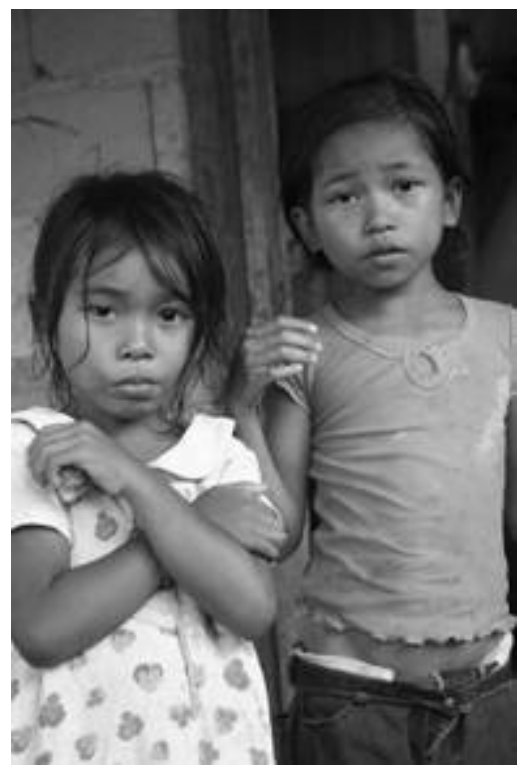

Figure 5.1 Siblings: K (left) is P's younger sister

significant sociolinguistic dynamism, thanks to patterns of social mobility and modernization associated with education and global cultural trends. These patterns of rich variation in modern Lao society, and the formally constrained registers of traditional ritual life, are set aside here, but will constitute an important later elaboration on the topic of Lao person reference.

\subsubsection{Ethnographic background to the hierarchical stance in Lao social organization}

The vagaries of Lao person reference are one reflex of a strong streak of verticality or 'authority ranking' (Fiske 1992) in Lao socio-cultural organization. At the kernel of this hierarchical system is a special attention to the distinction in age between siblings, and a range of ways in which this is reflected in social practice, both linguistic and otherwise. To illustrate, consider a number of linguistic and ethnographic observations of some of the consequences of relative age status between the two girls in Figure 5.1.

A. These two sisters use different terms to describe their relationship to the other: There is no word in Lao meaning 'sister', the available kin terms obligatorily marking difference of age: K is P's nò̀ng4 'younger sibling', 
$\mathrm{P}$ is K's qùaj4 'elder sister'. (For explication of the many ways in which this sibling age distinction is further reflected in the semantics of the Lao system of kin terminology, see Enfield 2005: 54-6.)

B. Elder and younger siblings normally use different pronouns with each other, the elder using the bare, 'non respect' forms kuu3 'I' and mùng2 'you', the younger using polite forms khòoj5 'I' and caw4 'you'. (See below and Enfield 2006b; note that immediately adjacent siblings are sometimes exceptional in this regard, reciprocally using the non-respect pronominal forms.)

C. Elder and younger siblings use distinct formats for addressing and referring to the other by name, adding different social - hierarchical name titles in accordance with the level of 'respect' displayed by the choice of pronominal in (B), above: Here, the elder will prefix the younger's name with the 'female non-respect' form qiil-, the younger will prefix the elder's name with the 'elder sister' form qùaj4- (see below sections for elaboration).

D. Elder and younger siblings have different rights and responsibilities in domestic life. Elder siblings are often directly responsible for the care of younger siblings, especially at a very young age (from, say, 1-4). At the same time, as soon as younger siblings become 'useful', an elder sibling may freely delegate the younger to carry out chores.

E. If the younger of these two girls is married before the elder, she is required to perform a ritual of atonement, apparently for making her appear a spinster.

F. There are constraints on marriage defined in terms of sibling order (see Enfield 2005 for details): If the elder is married, the younger may marry her husband's brother, but only if he is the younger brother, not if he is the older; If the children or grandchildren of these two girls want to marry (i.e., as cousins or second-cousins), it is permissible only if the male of the pair is in the elder girl's line - that is, the male should be on the 'higher' line, irrespective of absolute age.

G. The first-born child gains a special prominence via the practice of teknonymy (Lévi-Strauss 1969: 349, see below), by which the parents come to be known by his or her name.

$\mathrm{H}$. The last-born is treated differently in that they traditionally inherit the family home and much of the land, and are expected to live with the parents in their old age.

This outline of some practices associated with the simple distinction in age/ order between siblings should suffice to give a sense of the degree of importance in Lao social practice of kin-derived hierarchical order. Let's now turn to a more focused consideration of the linguistic practices implicated by this. I 
begin with a sketch of two basic means for referring to people: pronouns and names.

\subsubsection{Pronouns}

True pronouns in Lao encode four levels of politeness, in each of the first-, second- and third-person singular forms (cf. Enfield 2006b). The system is typical in mainland Southeast Asia (Cooke 1968). ${ }^{2}$

Fewer distinctions are made in the plural. None of the forms encode the sex of the referent. One way of thinking about the distinctions encoded here is of the more polite second-person forms as successively raising the conceived status of the addressee, while the more polite first-person forms successively lower the conceived status of the speaker (Brown and Levinson 1987). This is reflected, for example, in the etymology of the polite forms khòj5 'I' (elsewhere 'slave') and caw4 'you' (elsewhere 'lord').

Some nominals such as occupational and kin titles function as pronouns (i.e., are used for tracking reference through discourse in successive non-initial mentions), yet without shifting their reference according to who is speaking. In the following example, the speaker refers to a (present) third person. The referent is not the speaker's actual uncle.

Example (1) (Woman is talking about an elder man.)

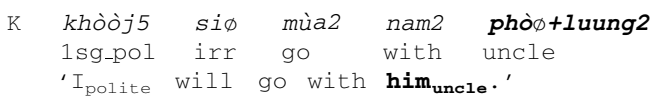

(See Table 5.1.) The next example shows kin terms where one may expect pronouns, but again unlike pronouns, they do not change their reference in a given context when a different speaker utters them. Among courting youths, the boy is qaaj4 'older brother', whether it is him or the girl who utters the word (mutatis mutandis for nò̀ng4 'younger sibling', the girl): ${ }^{3}$

2 Transcription of Lao here follows International Phonetic Association convention, except for $q$ (glottal stop), $\tilde{n}$ (palatal nasal), $n g$ (velar nasal), $\hat{e}$ (mid front vowel), $\grave{e}$ (low front vowel), $\grave{u}$ (high back unrounded vowel), $\grave{o}$ (low back vowel). There is no standard romanization of Lao. Lexical tones are marked by numerals at end of each syllable: 1 (mid level), 2 (high rising), 3 (low rising), 4 (high falling), 5 (low falling), $\varnothing$ (unstressed). Glosses of examples are according to the following conventions: 1/2/3 (first/second/third person), DEM (demonstrative), DIST (distal), F (female), FOC (focus), IRR (irrealis), M (male), PCL (particle), POL (polite), RESP (respect), SG (singular), TPC (topic); (n.n) means there is n.n seconds of silence. Kinship notation follows Parkin (1997:9): F (father), M (mother), B (brother), Z (sister), S (son), D (daughter), H (husband), W (wife), P (parent), G (sibling), E (spouse), C (child), e (elder), y (younger).

3 This pattern of use of kin terms among courtiers is distinctly old-fashioned/rustic, and is going out of date among the urban youth. 
Table 5.1 Some commonly used Lao pronouns ${ }^{\mathrm{i}}$

\begin{tabular}{lllll}
\hline \hline & & 1st & 2nd & 3rd \\
\hline Singular & Bare & kuu3 & mùng2 & man2 \\
& Familiar & haw2 & too3 & laaw2 \\
& Polite & khòoj5 & caw4 & phen1 \\
& Formal & khaa5-phacaw4 & thaan1 & thaan1 \\
\multirow{4}{*}{ Plural } & Bare & & suu3 & khaw3 \\
& polite & cu-haw2(incl.) & cu-caw4 & khacaw4 \\
& & cu-khòoj5(excl.) & \\
\hline \hline
\end{tabular}

${ }^{\mathrm{i}}$ These are the most common pronouns. Further pronouns, and further special uses of the pronouns in Table 5.1, are beyond the scope of this discussion.

Example (2) (Two youngsters courting.)

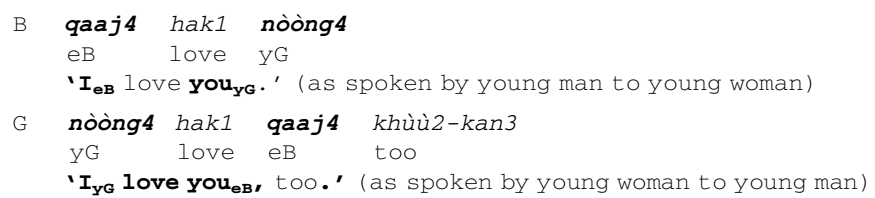

It is normal in many contexts for such use of kin terms to persist through a given stretch of interaction, where a kin term like qaaj4 ' $\mathrm{eB}$ ' will serve as the form for successive non-initial references - that is, functionally equivalent to pronouns in English discourse (Fox 1987).

\subsubsection{Lao names and name prefixes}

Lao full names typically have two components, a first name and a surname (e.g., Kaysone Phomvihan, Phoumi Vongvichit, Khamtai Siphandone). The surname is inherited from the father. These full names are largely derived from the Indic languages, Pali or Sanskrit. They are seldom used for person reference in informal conversation. Nicknames are normally used instead. These may be shortened from the person's full name (e.g., vaat5 from thongsavaat5 or thip1 from thipphacan3) or may be independently bestowed upon the person in infancy or childhood, typically on the basis of some attributed physical feature (e.g., tuj4 'fat', còoj1 'skinny', lèè 'blackened', nòoj4 'small'). A few full names are monosyllabic, and so are not in need of abbreviation for everyday use; for example, kèèw4, kuq2, mò̀n1. Most names may be used for both men and women. 
Teknonymy is common in Laos - the practice by which 'a person is called the father, mother, grandfather or grandmother, etc., of one of his descendents instead of by his own name' (Lévi-Strauss 1969:349). When a first child is born, Lao-speaking parents will come to be called by many as 'father/mother of X', where $X$ is the child's name. This means that many people effectively acquire a new proper name when their first child is named.

When Lao speakers refer to people using personal names, in the standard informal village setting, they attach a type of title prefix to the personal name. The prefix explicitly encodes the (classificatory) kin or other relative relation of the referent individual to the speaker. If the referent is someone 'not above' - that is, not (classificatorily) older than - the speaker, then a non-respect prefix is used. There are two such prefixes, that distinguish the sex of the referent. The non-respect female name prefix is qiil, the male prefix is bak2. These look 'downward'. Morphosyntactically, they are a type of nominal classification device (Enfield 2004). They occur as heads of nominal phrases (and cannot occur alone), taking a range of modifiers, including demonstratives, adjectives, relative clauses and names.

While the non-respect prefixes generalize across the variety of conceivable 'downward-looking' relations (e.g., younger sibling, nephew, niece, child, grandchild), referents who are 'above' the speaker receive a prefix from a differentiated range of name prefixes, selected from the everyday kinship vocabulary (each form also having independent use as a full kin term) (see Table 5.2)

In addition, a number of name prefixes denote the social rank of a referent (not relative to speaker), for example, as determined by monastic education (see Table 5.3)

So, if a speaker wants to refer to a thirty-year-old man called Phêt1, the prefix selected will depend, among other things, on the relative social position of the man to the speaker $(3 \mathrm{a}-\mathrm{c})$ or the man's absolute status $(3 \mathrm{~d})$ :

\section{Example (3)}

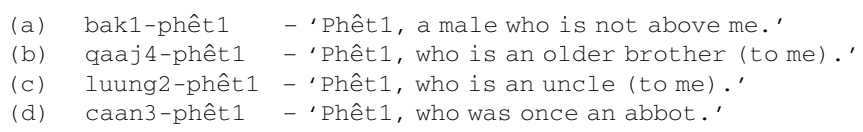

\subsubsection{Default formulations of initial references to persons in Lao conversation}

In informal, familiar, village conversation in Lao, the default way to formulate initial reference to a person is to use the person's familiar name prefixed by the 
Table 5.2 Some kin-related ('upward') name prefixes

\begin{tabular}{lll}
\hline \hline & Female & Male \\
\hline Elder sibling & qùaj4- & qaaj4- \\
Parent's elder sibling & paa4- & luung2- \\
Mother's younger sibling & saaw3- & baaw1- \\
Father's younger sibling & qaa3- & qaaw3- \\
Parent's parent & phò-tuu4- & mè-tuu4- \\
\hline \hline
\end{tabular}

Table 5.3 Some rank/role denoting name prefixes

\begin{tabular}{ll}
\hline \hline Ex-novice (novice=junior monk) & siang2- \\
Ex-monk & thit1- \\
Ex-abbot & caan3- \\
Monk/teacher & qacaan3- \\
\hline
\end{tabular}

form that appropriately denotes the referent's social position relative to the speaker (as in $3 \mathrm{a}-\mathrm{c}$ ). As long as the prefix is of the appropriate level, the 'prefix-plus-name' formulation is the default option, and its deployment in interaction will pass without special notice.

The following example is from a conversation between two men, Kou (K) and Xai (X), in Doune Ian, a village with about 500 inhabitants located some $35 \mathrm{~km}$ northeast of the Lao capital, Vientiane. Kou is about sixty years old, Xai about forty. Kou is a contemporary of Xai's father, and is Xai's classificatory luung2 'elder uncle' (PeB). Xai is a bit older than Kou's eldest child. The example features an initial reference by Xai to his own younger brother (line 1), followed by an initial reference by Kou to another man of around Xai's age (line 2). The speakers are both older than, thus 'above', both the referent individuals. Accordingly, these speakers formulate their references to these 'lower males' by attaching the non-respect male prefix bak2 to the referents' names:

Example (4) (Xai and Kou are discussing possible routes for a driving trip planned for later that day.)

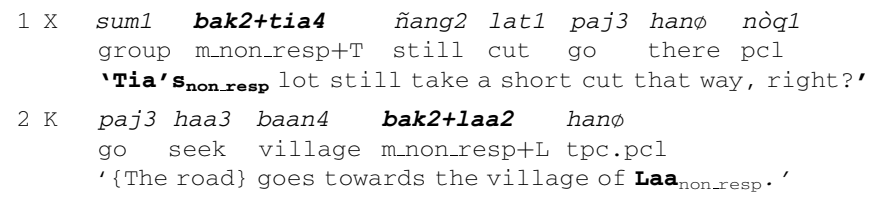


Nothing in this stretch of conversation indicates that these explicit indications of 'non-respect' are in any way foregrounded in the main communicative business.

The next example is from a conversation that takes place during a visit by Kêêt and Kaap, a middle-aged couple, to the house of an older couple (Grandma and Grandpa) in a somewhat remote village about $50 \mathrm{~km}$ outside of Vientiane. Grandpa is recuperating from injuries sustained when he fell off the front steps of his house some days earlier. Kêêt and Kaap live in the city of Vientiane, near Grandma and Grandpa's son Mòòn. In this example, Kêêt reports to Grandma and Grandpa that she had heard the news of Grandpa's accident from Grandpa's son Mò̀n. Kêêt's formulation of the initial reference by name to Mòòn is prefixed by the male non-respect form bak2- (line 1). In line 2, Kêêt makes subsequent reference to the same individual, using a thirdperson singular pronoun, here the 'lowest' available (see Table 5.1). This is appropriate to the register in which the prefix bak2-occurs. This is a Lao manifestation of a classic sequence of form-occasion alignment (Schegloff 1996a; cf. introduction to this volume, Fox 1987): that is, initial reference form (full noun phrase) on initial occasion of reference (line 1), subsequent reference form (reduced pronoun) on subsequent occasion of reference (line 2).

Example (5) (Speaker reports to Mòòn's parents that she'd heard news from Mòn in the town.)

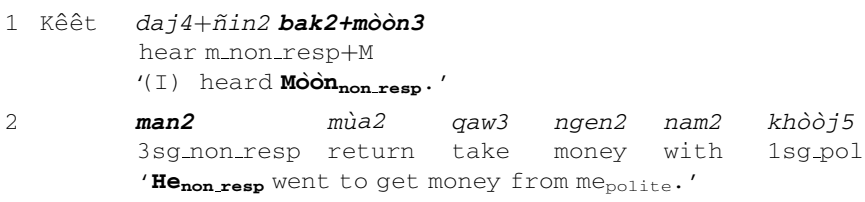

The precise formulations employed in the two explicit references to Mòn, in boldface, are defined by the social relationship that pertains between him and the speaker.

The examples so far have featured overt marking of names using non-respect 'lower' prefixes. The next example illustrates 'upward' directed marking of person reference. Here, a woman answers a question as to the location of somebody's house. She formulates it as being near the house of an older woman called Teng. The name is prefixed by the term paa4 'older aunty' (although the referent is the speaker's classificatory aunt, not her actual aunt):

Example (6) (Speaker describing the location of another village.)

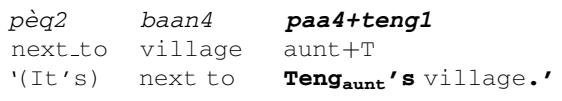


5.1.4.1 Maintaining differential perspectives to a single referent in conversation Since standard formulations of initial person reference include overt marking of social relation on a vertically oriented hierarchy, it often happens that a referent individual occupies a social position BETWEEN two interlocutors. The result is that two speakers will use different formulations for a single referent, not only for initial mention, but persistently, in successive mentions throughout a conversation.

The referent in the following example is a man named Khamlaa. Reference to Khamlaa occurs in a conversation between a group of six or so women who are chatting during a break in a reed-mat weaving session. The first reference to him is by Jot, who is younger than Khamlaa. Accordingly, Jot formulates her reference by prefixing Khamlaa's name with qaaj4 'older brother'. In line 2, one of Jot's interlocutors, an older woman named Mòòn, does a partial repeat of Jot's utterance (in line 2). Mòon is older than the referent individual Khamlaa, and accordingly her partial repeat of Jot's utterance in line 2 features a replacement of the 'older brother' prefix with the non-respect male prefix bak2-. This looks at first glance like an 'embedded correction' (Jefferson 1987), that is where one speaker replaces some component of another speaker's previous formulation, thereby achieving a 'correction', yet without having to disrupt the progressivity of the course of interaction. The difference here is that the 'replacement' is not treated as a correction. This differential formulation for person reference is maintained in subsequent occasions of reference in the sequence: In lines 4 and 9, Mòon refers to Khamlaa using the bare (lowest) third-person pronoun man2 (cf. Example (5)), while in line 7 , Jot refers to him using the familiar level third-person singular form laaw2, mirroring the two speakers' choice of prefix in formulating initial references.

None of these choices raises an eyebrow. They are pragmatically unmarked. Yet by their formal/semantic marking, they accurately respect differential relations of social height between each speaker and the referent individual. The ongoing different type of reference might be expected to create disaffiliation among the speakers involved: For example, if I persist in calling him Johnny while you persist with John, a discomfort may arise that causes one of us to change and accommodate to the other. In this context in Lao, no such discomfort arises. A possible conclusion is that correct social positioning outranks any potential inference of disaffiliation due to contrasting formulation (i.e., by creating a perception of unwillingness to accommodate and settle on a common way of saying it). 
Example (7) (Speakers are taking a break from weaving and are chatting. They have just noticed a car driving through the village.)

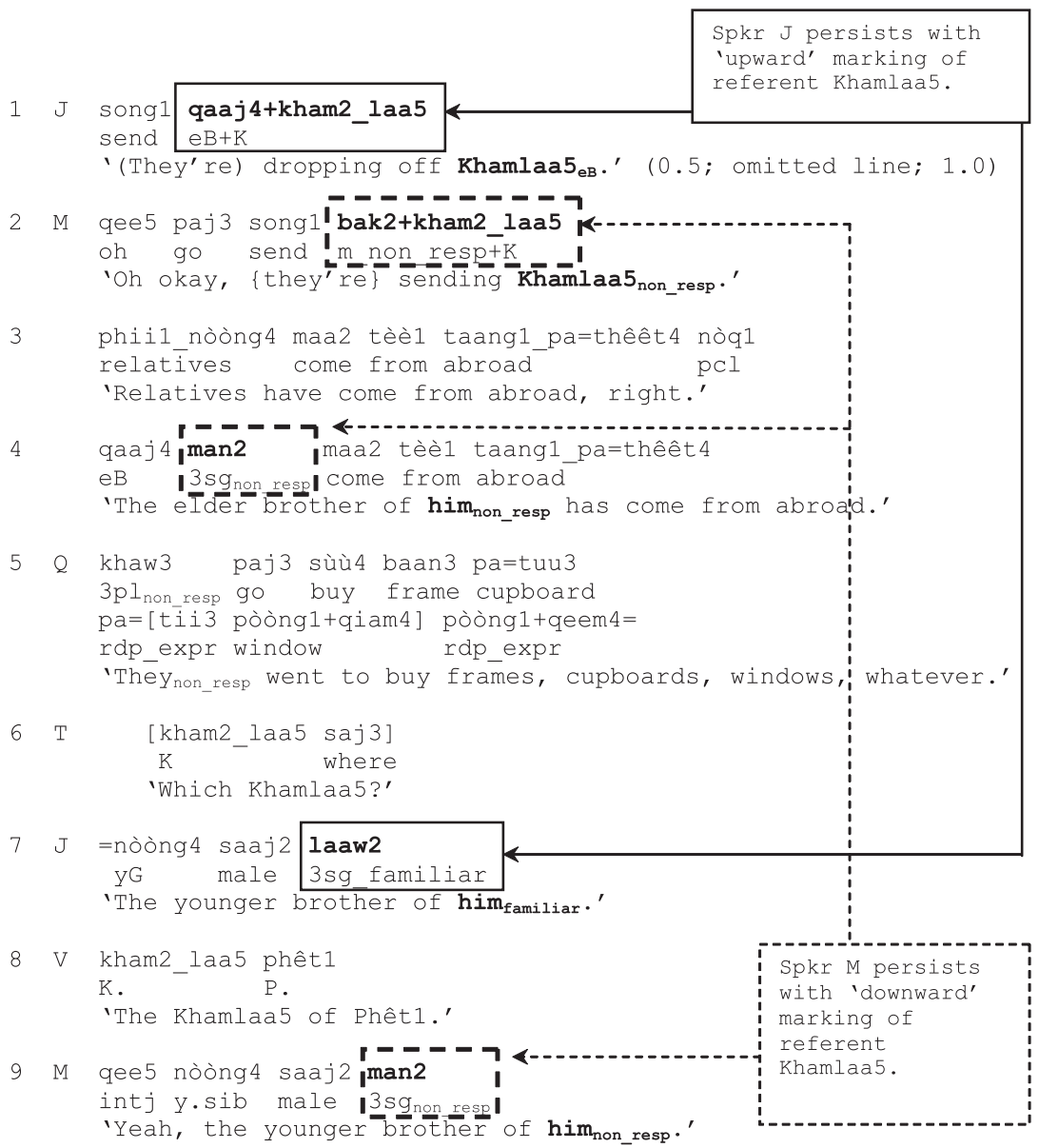

\subsubsection{Pragmatically marked formulations of initial references to persons}

The previous section described default, pragmatically unmarked referential formulations. We now consider some departures from these norms, before returning to the defaults and the question of what they might be communicating beyond mere reference. We consider cases in which speakers formulate initial references to persons in pragmatically marked ways, thereby 
drawing attention to the manner of formulation itself for some interactional purpose (Schegloff, this volume; Stivers, this volume).

5.1.5.1 Pragmatically marked selection of prefix One type of departure from the norm in Lao person reference is to observe the prefix-plus-name format but select a prefix that does not conform with the social relations pertaining. The following example is from the scene in which Kêêt and Kaap are visiting Grandma and Grandpa. In this example, Kêêt makes an initial reference to her own younger sister Daaw. Owing to the inherent downwardness of the relationship between an older and younger sibling, Kêêt's reference to Daaw would normally be marked by the non-respect female prefix qiil-: that is, Daaw is referred to as qiil-daaw3. However, the present instance calls for restraint. The purpose of the utterance in which Kêêt first mentions Daaw is to move into discussion of the main business of Kêêt's visit to Grandpa and Grandma. It concerns the donation of money by Daaw (who lives abroad), for renovations to the temple in Grandma and Grandpa's village. (Grandma and Grandpa are Daaw's parents-in-law; i.e., one of their sons is married to Daaw.) Kêêt's reference to Daaw is prefixed not by the expected non-respect prefix qiil- but by saaw3 'MyZ', an 'upwards' kin term that technically refers to the younger sister of one's mother.

Example (8) (Referent is speaker's younger sister, normally would use non-respect prefix qiil-)

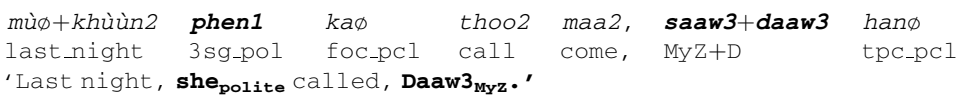

The third-person pronoun phenl that comes before the prefix-plus-name mention saaw3 daaw3 in the same sentence is a respect form (see Table 5.1). This higher reference is pragmatically marked for this combination of speaker and referent individual. Kêêt would normally use the non-respect third-person singular pronoun man2 to refer to Daaw.

The formulation of this reference to Daaw, the younger sister and daughterin-law, is pragmatically marked, signalling that the speaker is doing something more with this utterance than merely establishing reference to this person. The content of the marked formulation provides the information needed to figure out just what this special action is (Stivers, this volume). By referring to her own younger sister Daaw as 'mother's younger sister', Kêêt both casts herself in a lower-than-normal position (i.e., as niece), and casts the referent in a higher-than-normal position (i.e., as aunt). While pragmatically unmarked references presuppose the relations referred to, a marked usage such as this is akin to a creative indexical (Kockelman 2005); Silverstein 1976), introducing 
a new (not previously given) type of relation to the referent by virtue of its occasion of use.

In the next case, Xai makes reference to a man named Saaj, using the 'older brother' prefix (line 4). The other speaker, Kou, is Saaj's older brother, and accordingly Kou refers to Saaj with the non-respect prefix (line 6). However, since Kou is Xai's 'older uncle', Saaj is too 'high' to be an 'older brother' to Xai. It appears in line 4 that Xai is strategically using this person reference as a means to raise his own rank, pulling himself up in position. This move is entirely in line with his currently upwardly mobile status in life (he is about fourty years old, a successful small-time entrepreneur, eldest son of the present village chief).

Example (9) (Referent is Kou's younger brother; Kou is Xai's 'FeB'; thus, referent is too high to be Xai's 'eB'. Xai is upwardly mobile.)

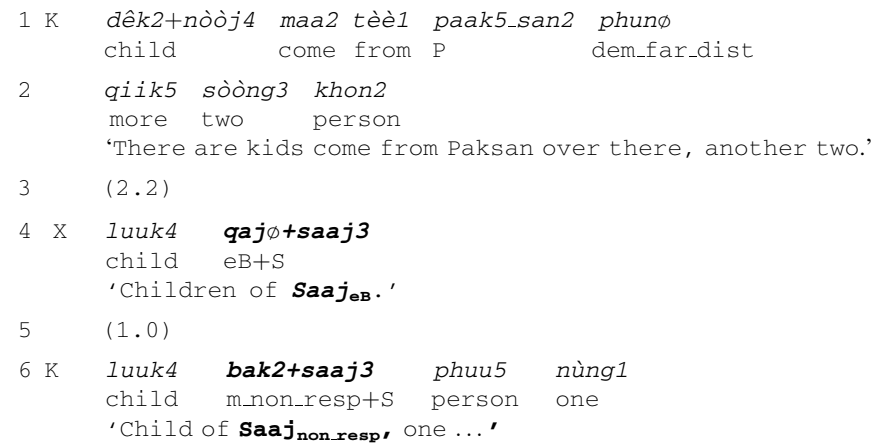

In the pragmatically marked formulations discussed in this section, speakers creatively manipulate the system to derive pragmatic effects concerning position within the social hierarchy, in one case for purposes of politeness (Example 9), in one case for Machiavellian purposes (Example 10; cf. Bloch 1971 on the notion of 'tactical' uses of kin terminology). Such examples are typical in Lao conversation.

5.1.5.2 Omission of prefix A different type of departure from norms in Lao person reference is to avoid altogether using the prefix-plus-name formulation, and instead using the person's name alone. In the following example, Kou refers to Nick, a foreigner and guest, who is present in the context (holding the video camera). Omission of the prefix signals politeness, derivable by inference (i.e., whereby omission of explicit marking of the expected 'non-respect' form implies the intention to communicate respect). The only appropriate prefix here is the non-respect prefix bak2-, as determined by the 
age difference. ${ }^{4}$ But the speaker refrains from using it. Thus, if the only available prefix clashes with the 'high' status of the referent (i.e., where status over-rides age, by virtue of being a present guest), a safe thing to do is to drop the prefix.

Example (10) (Kou [m, sixty-five years] is teasing an unmarried young woman; referent Nick is present.)

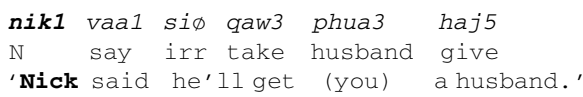

When Nick is out of earshot, Kou would normally refer to him using the nonrespect prefix, that is, as bak2-nikl. The respect inferable from the omission of an expected 'downward' referring prefix in (10) contrasts with what would be standard in informal village interaction. The following example shows that a local of the village gets the non-respect prefix in a comparable situation to (10) - that is, when he is present while being referred to as a third person:

Example (11) (Mek, Kou, Xai, and Nick are standing around waiting; Mek knows that Kou and Nick are going on a trip. He addresses Kou. Mek is the oldest of the group. The referent individual, Xai, is present.)

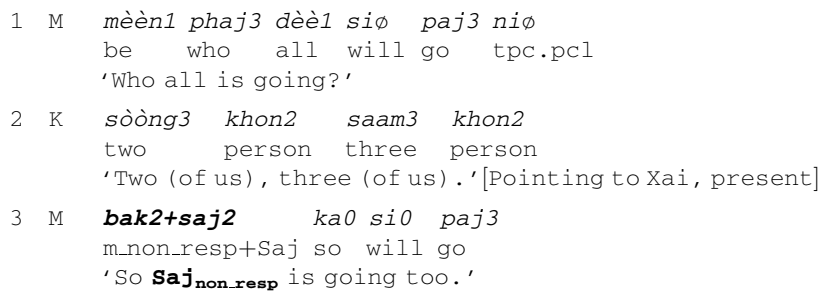

There are classes of situation in which the 'zero prefix' solution is standard. One such situation concerns newlyweds, that is after they should no longer be using the 'older brother' and 'younger sister' prefixes (see (2)), and before they are able to use teknonyms (since they don't have children yet). This operates by a logic of refraining from undesirable options, and perhaps works by means of depriving the liminal pair of any true default option at all - that is, all references will be pragmatically marked. The omission of

4 Another possibility is that Kou could transpose, and say qaaj4-nikl 'elder brother Nick', choosing the term that his interlocutor would have used (i.e., assuming the perspective of his interlocutor). 
title prefix to a name works by not overtly putting the referent into any kind of social position relative to the speaker. Since to omit such reference is pragmatically marked, the effect of such omission is to be heard as overtly refraining from specifying the referent as socially positioned relative to oneself.

\subsubsection{Summary: the Lao person-reference system}

The Lao person-reference system standardly requires explicit attention to relative social positioning among people in the village setting. Pragmatically marked forms of person reference in Lao exploit the default status of the prefixplus-name format, where the prefix situates the referent in terms of social structure (calculated as kinship, at least for 'upward-looking' references) relative to the speaker. The pragmatically marked uses derive their effects either by use of unexpected selection of prefix level (either creatively raising or lowering the referent, with the available implication of thereby lowering or raising the self), or by omitting any prefix and refraining from any explicit statement of social position of referent to speaker.

In the Lao system, while the default manner of formulation for person reference produces expressions that are by definition unmarked pragmatically, these expressions are explicitly marked, formally and semantically. We now consider implications of this for a general theory of person reference, and for associated issues of social action by means of the deployment of symbolic code (language) in interaction.

\subsection{Default formulations and what they do}

\subsubsection{How default formulations work}

In conversation, the continuous flow of collaborative action puts a heavy load on an individual's cognitive processing. It is reasonable to expect that this load will be minimized where possible (Gigerenzer et al. 1999; Zipf 1949). One type of solution is to automatize processing (in both formulation and interpretation) by assuming defaults. Current work in developmental and evolutionary psychology suggests that we are cognitively disposed to adopt locally learned defaults as a matter of principle (Boyd and Richerson 2005; Gergely and Csibra 2006; Simon 1990). Unquestioningly adopting convention requires a little trust, but is easy, and makes good sense statistically (Gigerenzer et al. 1999; Richerson and Boyd 2005). Culture is the supplier of such default conventions for all manner of communicative behaviour and background (Enfield 2000; Levinson 1995). Hence, we come to communication armed with maxim-like common expectations (Grice 1989), shared bases upon which to apply interpretative strategies to what's being perceptibly said and done in interaction. 
Whatever a person says, they have to say it in some way. If we had to ask ourselves upon hearing every piece of every utterance, 'Why is this person saying that IN THAT WAY?', we would unnecessarily (and, probably, unbearably) overburden our inferential and interpretative processing. ${ }^{5}$ Default, publicly shared, common assumptions about how people typically do and say things provide interpretative channels along which we may travel with minimal cognitive effort. The default is a device by which we can routinely suppress our attention to manner of formulation, and against which we may recognize when something is being done in an unusual way (being then able to ask ourselves why that might be). When a communicative action is done in the default way, the MANNER of action itself must at some level be recognizable as irrelevant to our task of interpreting what's 'being done' by that communicative action. It's against the background of this default that we may recognize the departures, and only THEN actively apply our rational interpretation: WHY THA T NOW ? ${ }^{6}$ (Grice 1989, Schegloff 1996a, b) Further, to calculate what's being done, it's not just a matter of 'why that?' but also of 'why not the usual'?; see introduction, and Stivers, this volume. In short, the special role of defaults for communicative formulation is that they allow us to hear what people are saying without always having to wonder why they are saying it LIKE THAT and not in some other way. This disattention is the basis of Schegloff's (1996a) claim that defaults do not, and by definition cannot, 'do work' in social interactional terms.

\subsubsection{How defaults are disarmed in conversation analysis by a Members-Only Filter}

The defaults argument just outlined is implied by Grice $(1975,1989)$ and descendents (e.g., Levinson 2000), and is standardly employed in Conversation Analysis (although it is never discussed there in terms of economy of cognitive processing). Schegloff's (1996a: 439) argument that default personreference formulations make no contribution beyond mere referring is in line with a fundamental methodological and analytic tool in Conversation Analysis that I shall call a Members-Only Filter. Members are those people participating

5 The relative arbitrariness of the form-meaning mapping in spoken language is facilitated by the phonological medium's poor fit for iconic and indexical relations to complex ideas. This low motivatedness of the modality is what allows us to best disattend to the specifics of manner of formulation in speech. The greater the potential for iconicity in the modality of representation, the harder it is to bracket out the implications of form selection, since if there is an easily conceivable link from form to meaning, then it may be readily seen as a motivation. This is an issue for comparative work between signed and spoken modalities of language (cf. Kendon 1988; Taub 2001; Emmorey 2002; Meier et al. 2002; Liddell 2003, inter alia).

6 This is exactly analogous to the finding of Gergely et al. (2002) about the earliest signs of rationality in infants. This kind of reasoning is fundamental to our cognition, and is well in place before we have language or other complex interactional skills (Gergely and Csibra 2006). See also Sperber and Wilson (1995). 
in interactions that comprise the data set for analysis (Garfinkel 1967; Sacks 1992). This filter allows the radar screen to display only those actions that are both 'available to' and 'oriented to by' the interactants involved.

Methodologically, the Members-Only Filter is intended to determine for the analyst what will, and what will not, be counted for consideration as relevant to the interaction and its analysis. For the conversation analyst looking to identify social actions being accomplished in interaction, this filter lets through only those candidate actions that afford 'some demonstration that the interlocutors in the data being examined have understood the utterances (or other conduct) in question to be possibly doing the proposed action(s) or that they are oriented to that possibility - a demonstration ordinarily grounded in the interlocutors' subsequent talk or conduct' (Schegloff 1996b: 172). These interpretative conclusions and the social actions they recognize must be 'available in the talk' (Heritage and Atkinson 1984: 8 - 9; cf.Sacks 1992; Schegloff and Sacks 1973). But what exactly does it mean for something to be 'available in the talk, available for inspection' (by interactants and analysts alike)? In one sense of available - let's call it formally available - something is explicitly coded in communicative behaviour. It can be pointed to. ${ }^{7}$ An example is the system of Lao kin prefixes described in this chapter. In a second sense of available - let's call it contextually available - something is accessible by being in the shared common ground (Clark 1996). For example, the commonly assumed access to linguistic paradigmatic structures allows us to make inferences based on what has NOT been said. If a speaker uses a $\mathrm{T}$ pronoun form when a $\mathrm{V}$ form was openly appropriate, it is FORMALLY available that the speaker chose the $\mathrm{T}$ form. In addition, it is CONTEXTUALLY available that the speaker COULD HAVE chosen the V form, and thus that they CHOSE NOT тO use the $\mathrm{V}$ form. This kind of availability directs your attention to what is MISSING, a critical notion in both Gricean pragmatics and conversation analysis. For a communicative action to be 'officially absent' (Schegloff 1968: 1083), hearers need to have stored what the contextually equivalent (i.e., paradigmatically related) options at that moment of inaction might have been. Such presumed or inferred pieces of meaning are contextually available, but not literally present in the talk, that is, not formally available.

But however we define it, availability alone isn't enough for the MembersOnly Filter. Even when something is explicitly available in the talk (such as the hierarchical differentiation of social relations encoded in Lao person-reference title prefixes), if interactants aren't also demonstrably 'oriented to' it - that is, displaying some kind of awareness, attention, recognition - it is said to be

7 Sometimes people will recognize it when it is pointed out, and find it so obvious that pointing it out at all is odd; for example, that the word table refers to a table. In other cases, they might not have noticed or thought about it before its being pointed out; for example, that Cinderella (availably!) incorporates the word cinder, indexing her line of work. 
irrelevant to the organization of the interaction, and therefore to its description and analysis. ${ }^{8}$ In the case of person reference, default formulations are said to be doing nothing but referring because they are not explicitly 'oriented to' by participants. In this view, it's not enough that the content in question is merely made available.

\subsubsection{What remains unseen depends on where your blind spot is}

In the Lao system of person reference, while overt specifications of kinship and other hierarchical social relations are unmarked or default in pragmatic terms, they are overtly marked both formally and semantically. These markings make explicit a person's hierarchical position relative to others in the social network, an important principle in Lao speakers' cultural understanding of personhood and society. A Members-Only Filter would reject any claim that speakers are 'doing' anything in social - interactional terms by using these socially hierarchical forms, on the grounds of a lack of 'orientation'.

Kitzinger (2005) delivers an important challenge to this stance in a study of verbal references to sexual relationships in English conversation (see also Land and Kitzinger 2005). On the one hand, in conversation, gay and lesbian speakers are typically unable to make simple references to their same-sex partners (e.g., as revealed by gender-specificity in English names, pronouns, etc.) without either being taken to be ('oriented to as') topicalizing their own sexuality, or resulting in an addressee topicalizing it. In a telephone conversation between Janice and a car insurance salesman (Land and Kitzinger 2005: 396-8), Janice says I'm wanting insurance for um two named drivers self and spouse, which a few turns later is glossed by the insurance salesman as you said you'd like to insure your husband to drive the car. Here, spouse is taken to have meant 'husband'. Janice is required momentarily to delay the conversation's progress with a correction It's not my husband it's my wife, but without delay gets back to the business of the call. A short time after, while waiting for a response from his computer system, the insurance man topicalizes Janice's same-sex marriage, resulting in an extended sequence of apologies and remarks on the heterosexist assumption. By contrast, heterosexual speakers run little risk of their sexuality being foregrounded when they reveal it to interlocutors in exactly the same simple ways (e.g., gender of names, pronouns, words like wife). They make public their heterosexuality entirely in passing, as

${ }^{8}$ In semiotic terms, the conversation analyst insists on a perceptible interpretant (i.e., a relevant response to the sign that may also be taken to be a response to the sign's meaning, providing evidence for that meaning - cf. Kockelman 2005; Peirce 1965/1932). The semanticist is looking for an object (in the Peircean sense; i.e., the meaning, in cognitive or abstract terms): as long as he has a sign (e.g., an identifiable word), the semanticist is happy to supply his own interpretant (or bracket the interpretant altogether). 
'a commonplace and taken-for-granted feature of social interaction' (Kitzinger 2005: 259). Thus, if a woman states My husband isn't very well during a call to a doctor's office (Land and Kitzinger 2005:388, citing Drew 2006), she is unlikely to be taken to be (or 'oriented to as') topicalizing her sexual orientation, although she is nevertheless explicitly making it available.

In concluding her discussion on the distinct UN-remarkability of speakers' revealing their heterosexuality in English, Kitzinger (2005: 259) writes: 'As analysts, we might want not to take a member's perspective on this but rather to treat the interactant's everyday world as problematic ... [W]e might ask what is happening when nothing special is happening: ... when presumed ordinary experiences are treated as ordinary - what is happening THEN, how is THAT done, and what kind of a world must we be living in that these things run off smoothly?'

There is a clear parallel between the apparent invisibility of the heterosexist assumption in English person reference (associated with gender of pronouns, gender-specificity of names, relational terms like husband and wife, among further things) and the apparent invisibility of social hierarchy in Lao personreference kin titles. For some things to go so unquestioned that their explicit display is unnoticed (but not by all!), we have to be living in a kind of world where people assume just that thing to be the default in interaction. And it is not a matter of what people actually assume (e.g., that people are heterosexual), but of what they assume will generically be assumed by others (Enfield 2002: 16 - 17). Adopting the attitude of a 'generalized other' is what situates the individual in society and culture (Mead 1934). At the micro-level this matters only as a principle of agreement for communicative pragmatics. When we ask how a particular interpretative convention has come about socio-historically, we may well ask WHY THAT? Why is THAT the default and not something else? In the case of Lao kin prefixes, the encoded meaning reflects the conventional way of doing person reference. The social distinction encoded in the choice of marking reference to Khamlaa 5 with an 'older brother' or a 'younger brother' prefix (see Example (7)) is as invisible and unremarkable in default usages as would be the heterosexuality indexed by a woman's passing use of the term my husband in referring to her husband. ${ }^{9}$ When a Lao speaker

\footnotetext{
9 Any difference between cultures in this respect is not a difference in assumptions about the way of the world. That is, in both Laos and England, interactants are necessarily of different ages relative to each other. The difference between the cultures here is in what is habitually made explicit in talk. English speakers don't systematically make relative age/rank available in language. From a Lao perspective, we studiously AVOID it, perhaps like the way a lesbian may be perceived to avoid certain types of relationship references (Kitzinger 2005:258). One has to be a member of another culture to 'see' the social hierarchy so ubiquitous in Lao person reference (Whorf 1956) - I'm not aware of a stigmatized sub-culture among Lao speakers for which such social-hierarchical assumptions are problematic, but one could conceivably exist (e.g., among young people in a globalizing world).
} 
explicitly ENCODES such a relation in person reference, as described in this chapter, she might not be topicalizing it or drawing attention to it in the sense of making it the business of the utterance in which it is embedded. But she is nonetheless explicitly encoding it. This is reason enough to suspect that these encoded social relations are serving socially communicative ends. At the very least they convey a routine willingness to make key social relations public, thereby reasserting, reiterating, and reproducing these core cultural concerns. Such unceasing yet entirely automatized attention to accurate representation of hierarchical social relations in talk is a contribution all Lao-speaking individuals make to the stability of Lao (speaking) cultural values.

Consider what might be 'available yet not oriented to' in a more familiar person-reference system, English. The argument based on Lao data may seem straightforward, since the cultural value at hand is explicitly articulated; that is, hierarchical person reference based on asymmetrical kinship. To turn it around, the Lao system of person reference points to what is available yet automatized in the defaults of the English system. When Lao speakers make person references using a name with a kin prefix, they overtly encode that person's differential social position, publicly reproducing a specific cultural value - we're all at different positions on an unequal hierarchy - each time they do it. From the outside, we might see this as an obsession with hierarchy. Similarly, when English speakers make person references using a bare name, they not only achieve reference (i.e., convey to their addressee who it is they are talking about), but in addition they make available a virtual assertion of that person's NON-differential position, publicly reproducing a specific Anglo cultural value - we're all at the same level on an equal plane - each time they do it. From the outside, one might see this as an obsession with egalitarianism. This is confirmed in widely reported ethnographic observations on the social advantages and disadvantages of speaking English; for example, the ease of having one and only one second-person pronoun rather than being forced to choose between formal/distant and informal/intimate forms; the discomfort of having to call a superior by first name; the discomfort of hearing a subordinate call one by one's first name (cf. Brown and Gilman 1960; Wierzbicka 1992). ${ }^{10}$ While the Lao pattern is to overtly mark relative social status as a pragmatically unmarked way of referring to persons, English has no overt marking. The 'social statement' in the English person-reference system is contextually

10 And default person reference by name in English is no simple matter. Even among first names, there are multiple choices, with multiple meanings. We may not know in advance which of Fred, Frederick, Freddy or Jer, Jerrold, Jerry is the default or pragmatically unmarked form for a given configuration of speaker-hearer-referent. (Although we may know, for example, that Jer, Frederick and Freddy are systemically marked, while Jerrold, Fred, and Jerry are not; Wierzbicka 1992: 225ff, 303.) Furthermore, it may be argued that names fall into type classes, and as such have (at least connotative) meanings (see Introduction to this volume). 
available but not formally available. But the English system nonetheless embodies a cultural set of values concerning the social web of personal relations.

While I am arguing against the claim that a default person-reference formulation does literally 'nothing more than refer' (Schegloff 1996a), I acknowledge an important insight behind that idea. It clearly matters that pragmatically unmarked formulations pass in conversation without apparent notice or attention. How can I claim that there is some kind of 'work' being done by a distinction that apparently remains out of awareness? The answer lies in Sacks' observation, cited at the opening of this chapter, that it takes special work to appear so ordinary that one's manner of behaviour becomes literally unremarkable. A key observation made by Sapir, Whorf, and others investigating the relation between language, thought and culture was that much of language is outside our awareness (Jakobson 1957). This motivated Whorf's quest to study languages unlike those familiar to Anglo-European scholars. On the idea of a rule and our native conceptual access to it, Whorf wrote: 'Never having experienced anything in contrast to it, we cannot isolate it and formulate it as a rule until we so enlarge our experience and expand our base of reference that we encounter an interruption of its regularity' (1956: 209). If a group of people only saw blue, he suggested, 'they would hardly be able to formulate the rule that they only saw blue'. To be able to formulate a rule that they only saw blue, 'they would need exceptional moments in which they saw other colors' (1956: 209). Part of Whorf's insight was that we 'march in step' with distinctions that have a 'background character' in our own languages. '[O]ur psychic make-up is somehow adjusted to disregard whole realms of phenomena that are so all-pervasive as to be irrelevant to our daily lives and needs' (Whorf 1956: 210). This 'disregard' is another word for the Conversational Analysts' 'lack of orientation' (see above). Despite the message's availability, its apparent disregard by interactants is taken to mean that it should therefore be disregarded by the analyst as well. But as Whorf pointed out, as soon as pragmatically marked formulations are 'isolated against a background' they bring that otherwise disregarded background into view. And it is no less real. As Lucy (1992: 37) puts it, 'we de-automatize our own language categories by contrasting them with those of other languages.' Contrast reveals what we habitually fail to see in the most everyday phenomena. Something meaningful may be all the while available in the data, but may take some pointing out before it is noticed or registered or oriented to. When we think differently to the defaults (as, say, an egalitarian in hierarchical society, or a lesbian in straight society), even for a moment, the otherwise default becomes marked. Despite members' typical disattention to the routinely available in uneventful interaction, the analyst would be crazy to ignore it. Consider the Lao case. That an entire society of individuals agree to make an explicit distinction in fundamental social 
structure in every second spoken utterance yet find that very distinction utterly unremarkable is in itself deserving of special interest.

\subsection{Conclusion}

In any society, an individual will occupy a set of places in a highly structured constellation of social relationships. And any society will have its conventional ideologies about the nature and structure of that constellation. Accordingly, members of any society will follow fashion in expressing those ideologies and publicly displaying their adherence to them. When Lao speakers refer to persons in conversation, the normal pragmatically unmarked strategy is to employ a formally and semantically marked formulation, by which the mention of a person's name is accompanied by explicit statement of that person's position, relative to the speaker, within a hierarchical system of social relations. This pragmatically UN-marked formulation for person reference is explicitly doing more than simply achieving reference, but this sense of 'doing' need not be the 'foregrounded social action now' that the term sometimes implies (Schegloff 1996a). It's one of many things a person does by silently conforming, and the forms of conformity are, after all, not wholly arbitrary. Lao practices of person reference do the basic work of referring to people, and, in addition, they make explicit the social position of the referent individual relative to the speaker - above or below, and if above, then classificatorily related to the speaker - for example, as sibling, aunt/uncle or grandparent.

By the same argument, English speakers also unavoidably give off a stance on social relationships when employing the person-reference resources made available by the norms of the culture. How could any language give us a way out of this problem? I submit that it is not possible in any context to refer to persons without encoding, implying, or otherwise making available a stance towards social relationships that applies generally in the culture. Indeed, this is just what perpetuates their status as culturally generalized. Formulations of person reference in any language system may well pass without special treatment of the way they are formulated, but they may always do more than just achieve reference. They make publicly overt and thereby instantiate and stabilize cultural values about persons and their social relations.

\section{Acknowledgements}

I gratefully acknowledge the input of the other participants in the MPI Nijmegen Person Reference Workshop, especially Penny Brown, Rebecca Clift, Bill Hanks, John Heritage, Celia Kitzinger, Steve Levinson, John Lucy, 
Federico Rossano, JP de Ruiter, Manny Schegloff, Gunter Senft, Jack Sidnell and Tanya Stivers. I thank Grant Evans for much useful discussion in Vientiane, January 2006. I benefited from a last-minute chance to present these ideas in Helsinki in May 2006. I thank the Max Planck Society for its support of this work. 